\title{
Etude diagnostique des pratiques de friture du thon du mets «Garba» consommé dans la ville d'Abidjan (Côte d'Ivoire)
}

\author{
Massogbè DIABATE ${ }^{1,2}$, Joseph DOSSOU ${ }^{2 *}$, Daouda NIMAGA ${ }^{1}$, Albarin G. GBOGOURI ${ }^{1}$ \\ et Georges N. AMANI ${ }^{1}$
}

${ }^{1}$ Laboratoire de Biochimie alimentaire et de Technologies des Produits Tropicaux (LBATPT), Université Nangui Abrogoua, BP 801 Abidjan, Côte d'Ivoire.

${ }^{2}$ Laboratoire de Bio ingénierie des Procédés Alimentaires (LABIOPA), Université d'Abomey Calavi, BP 526 Cotonou, Bénin.

*Auteur correspondant; E-mail : jokdossou@yahoo.fr; Tél: 00229-97086266

\section{RESUME}

L'objectif de l'étude était d'évaluer l'impact des pratiques de friture du thon du mets «Garba» sur la qualité sanitaire de l'huile de friture. Les pratiques de friture et les taux en composés néoformés ou polaires des huiles ont été déterminés dans les restaurants des communes de Cocody, Yopougon et Abobo à Abidjan. L'étude a revélé que la vente de ce mets est une activité d'hommes $(94,9 \%)$ n'ayant aucune formation academique $(62,8 \%)$ pratiquant la friture dans les conditions variées. Trois groupes de vendeurs ont été identifiés selon les pratiques adoptées. Le $1^{\text {er }}$ groupe est caractérisé par une pratique utilisant des températures inférieures à $160{ }^{\circ} \mathrm{C}$ et les huiles contiennent des composés néoformés inférieurs à $25 \%$. Le $2^{\text {ème }}$ utilise des températures de friture comprises entre $160{ }^{\circ} \mathrm{C}$ et $180{ }^{\circ} \mathrm{C}$ avec des huiles réutilisées contenant 25 à $75 \%$ de composés néoformés. Le $3^{\text {ème }}$ groupe fait la friture à $180{ }^{\circ} \mathrm{C}$ et les huiles réutilisées contiennent $75 \%$. La majorité des huiles analysées contiennent des taux en composés néoformés supérieurs au taux seuil de $25 \%$ fixé par le décret français $\mathrm{n}^{\circ} 86-857$ du 18 juillet 1986. Il ressort de cette étude qu'il y a un risque d'atteinte à la qualité sanitaire de ce mets et par conséquent à la santé des consommateurs.

() 2018 International Formulae Group. All rights reserved.

Mots clés: Composés polaires, qualité sanitaire, alimentation de rue, Garba, thon.

\section{Diagnostic study of the practices frying tuna of "Garba" food consumed in Abidjan (Côte d'Ivoire)}

\begin{abstract}
The study aims to evaluate the impact of tuna frying practices on the quality deterioration frying oil of "Garba". The frying practices adopted and the rate of total polar materials the frying oils have been assessed in the restaurants of Cocody, Yopougon and Abobo in Abidjan. the study shown that the sale of "Garba" food is a men $(94,9 \%)$ activity having no academic training $(62,8 \%)$. This frying is done under various conditions. Three groups of sellers were identified according to the adopted frying practices. The first group is sellers characterized by frying at temperatures below $160^{\circ} \mathrm{C}$ and the oils contain total polar materials levels less than $25 \%$. The second group is sellers using temperatures between $160{ }^{\circ} \mathrm{C}$ and $180{ }^{\circ} \mathrm{C}$ with used oils with a compounding ratio between 25 and $75 \%$ total polar materials. The third group fried at $180{ }^{\circ} \mathrm{C}$ and the reused oils contained $75 \%$ total polar materials. The majority of the oils analyzed contain total polar materials higher
\end{abstract}


than $25 \%$ fixed by the french decree ${ }^{\circ} 86-857$ of July 18,1986 . This study shows that there is a risk of the quality oil of "Garba" and consequently the health of consumers.

(C) 2018 International Formulae Group. All rights reserved.

Keywords: Total polar materials, food safety, street food, Garba, tuna.

\section{INTRODUCTION}

En Afrique, le secteur de l'alimentation de rue a envahi les zones de forte activité économique et de forte concentration démographique (FAO, 2015). L'évolution du mode de vie (éloignement du lieu de travail, alimentation déstructurée...) a contribué à une grande consommation de ces aliments préparés généralement à base de produits locaux au pétit- déjeuner, au déjeuner et même au dîner (Amani et Kamenan, 2003).

En Côte d'Ivoire, le mets «Garba », aliment de rue préparé à base de produits locaux, est beaucoup consommé à tout moment de la journée. Dans la préparation de ce mets, il faut : de l'attiéké, du thon frit, du piment frais et selon les préférences, de la tomate, de l'oignon frais et de la mayonnaise, le tout relevé par le sel de table ou un bouillon culinaire (Tanoh, 2016; Konan et al., 2007). L'attiéké, couscous de manioc fermenté cuit à la vapeur, était à l'origine un plat de réjouissance des peuplades du littoral ivoirien avant de s'imposer comme un plat national (Ayawoyi et al., 2016). Il existe 3 types d'attiéké selon la taille, la texture des grains et l'origine ethnoculturelle de la production. L'attiéké agbodjama de couleur blanchâtre composé de gros ou petits grains, l'attiéké adjoukrou de couleur jaune composé d'un mélange de moyens et petits grains, et l'attiéké de «Garba» de couleur crème composé souvent d'un mélange de grains difficilement différenciables. Ce dernier type est de qualité inférieure par rapport aux deux précédents (Assanvo et al., 2002).

Le mets «Garba» ou attiéképoisson thon est, par excellence, un repas typiquement ivoirien, rapide, bon marché et prisé par toutes les franges de la population (Bassa et al., 2016). Depuis sa vulgarisation au début des années 90 par le courant musical zouglou en Côte d'Ivoire, on a assisté à Abidjan à une éclosion de gargotes (petites échoppes de rue) du mets qui se sont installées aux alentours des cités universitaires, des établissements scolaires, des zones industrielles, des centres d'affaires, des marchés et dans les quartiers (Heuberger, 2005).

Ces dernières années, cet aliment de rue fait face à une demande sans cesse croissante en milieu urbain et rural. Toutefois, les profits générés par les acteurs du secteur et l'inexistence de réglementation pour les aliments de rue en général semblent les conduire à développer une certaine stratégie visant à privilégier la réduction des coûts de production au détriment de la qualité. En ce sens, les pratiques adoptées pour la friture du thon notamment : l'utilisation d'équipements rudimentaires et obsolètes, le type, l'origine, la qualité et la surutilisation de l'huile lors de la friture à des températures très élevées pourraient en dégrader la qualité sanitaire. Ces pratiques favoriseraient une forte production de composés indésirables (néoformés ou polaires) dues aux nombreuses réactions chimiques dont l'oxydation, la polymérisation et l'hydrolyse qui se réalisent lors de la friture (Choe et Min, 2007). Ces composés néoformés ou polaires ingérés à une certaine dose peuvent s'avérer nuisibles pour la santé (Courtois et al., 2012). Csallany et al., 2015 ont souligné le risque alimentaire lié au chauffage et à la réutilisation d'huile à cause de la production de quelques composés cancérigènes, notamment les hydrocarbures, les acides gras libres, l'acrylamide, les peroxydes et les aldéhydes toxiques.

Malgré l'attrait économique et l'engouement suscité par la vente du mets «Garba» à Abidjan, aucune donnée scientifique n'a été trouvée sur cette activité qui reste encore informelle. Il convient donc de produire des données et des informations scientifiques sur la friture du thon et d'évaluer l'impact des pratiques sur la qualité sanitaire du mets 
«Garba». Plus spécifiquement, il s'agit de rechercher les différentes pratiques de friture et d'évaluer la teneur en composés néoformés ou polaires des huiles de friture réutilisées dans les gargotes de trois communes d'Abidjan.

\section{MATERIEL ET METHODES \\ Enquête sur les pratiques de friture des vendeurs du mets «Garba» \\ Echantillonnage}

L'étude s'est déroulée dans trois communes de la ville d'Abidjan, à savoir Cocody, Yopougon et Abobo. La sélection de ces communes se fonde sur le constat selon lequel toutes les classes sociales consomment le mets «Garba» et tient compte du niveau de prospérité tel que perçu par la population dans la ville d'abidjan : la commune de Cocody est habitée par les personnes aisées à haut niveau de revenu. Yopougon est une commune où habitent les personnes de classe moyenne. Abobo, commune populaire d'Abidjan, est majoritairement habitée par les personnes à faible revenu. Le choix des vendeurs de ce mets a été réalisé par un sondage à deux dégrés. Au premier dégré, la base de sondage est constituée de la liste des communes d'Abidjan, dont les données démographiques sont issues du Recensement Général de la Population et de l'Habitat (RGPH) de 2014 (Institut national de la statistique de Côte d'Ivoire, 2015). Au second dégré, 10 secteurs ont été sélectionnés de façon aléatoire dans chacune des communes, et ensuite 03 vendeurs du mets «Garba» ont été choisis par secteur selon la technique d'enquête non probabiliste dite «boule de neige».

\section{Collectes des données}

Les données collectées lors de cette enquête portent sur: l'identification du vendeur (classe d'âge, sexe, niveau d'instruction), les pratiques de friture (source d'énergie, nature de l'huile, lieu d'achat de l'huile, quantité d'huile par friture, proportion d'huile ajoutée, nombre de lots de poissons frits avant rajout d'huile, temps de friture, température de friture et taux en composés néoformés ou polaires, nommés aussi TPM (Total Polar Materials) produits dans les bains d'huiles. En dehors de la température de l'huile et du taux de composés néoformés qui ont été mesurés, les autres données ont été collectées à la suite des entretiens structurés. Le Tableau 1 présente ces différentes variables ainsi que leurs modalités.

\section{Détermination du taux de composés néoformes des huiles réutilisées de friture Matériel biologique}

Le matériel biologique utilisé dans ce volet était constitué d'huile végétale raffinée réutilisée pour la friture du thon échantillonnée chez les vendeurs du mets «Garba».

\section{Méthode de dosage des composés néoformés}

La méthode officielle de dosage de composés néoformés des huiles et graisses alimentaires fixée par l'arrêté du $1^{\mathrm{er}}$ octobre 1986 et reprise par la norme NF EN ISO 8420 , avec un kit de dosage a été appliquée. Ainsi, la détermination des teneurs en composés néoformés des huiles de friture a été effectuée à l'aide de l'appareil Testo $270\left(\mathrm{~N}^{\circ}\right.$ de série 20388792 de la firme polychimie, Allemagne). L'appareil a été plongé dans le bain d'huile chaud avant ajout des poissons ce qui a constitué le témoin et après la friture et le retrait d'un lot de thon de l'huile. Les taux de composés néoformés de l'huile ainsi que la température du bain d'huile ont été relevés sur l'écran du Testo - Analyseur.

\section{Traitement statistique des données}

Pour identifier et décrire les différentes pratiques adoptées par les vendeurs, une analyse des correspondances multiples a été réalisée. Les principales composantes ont été utilisées pour réaliser une classification hiérarchique des pratiques adoptées. Le choix du nombre d'axes est basé sur le principe de la variance maximale expliquée par les axes. Les modalités interprétées sont celles qui sont les mieux représentées et qui ont une forte contribution sur chaque axe. Elle est suivie d'une classification hiérarchique en vue de former des groupes homogènes de vendeurs du mets «Garba». Le module Factomine R du logiciel $\mathrm{R}$ version 2.15 .1 a été utilisé pour réaliser ces analyses. 
Tableau 1: Variables étudiées et leurs modalités.

\begin{tabular}{l|l}
\hline \multicolumn{1}{c|}{ Variables } & \multicolumn{1}{c}{ Modalités } \\
\hline Classes d'âges & Jeune (15 à 30 ans), Adulte (31 à 50 ans) \\
\hline Sexes & Masculin, Féminin \\
\hline Niveau d'instruction & Aucun, Primaire, Secondaire, Supérieure, Coranique \\
\hline Sources d'énergies & Bois, Charbon, Gaz \\
\hline Nature de l'huile & Palme raffinée, Inconnue \\
\hline Lieu d'achat de l'huile & Boutique, Marché, Supermarché, Marchand ambulant \\
\hline Quantité d'huile par friture & $<1 \mathrm{~L}, 1$ à 3L, 3 à 6L, 6 à 9L \\
\hline $\begin{array}{l}\text { Nombre d'utilisation de l'huile } \\
\text { avant rajout d'huile neuve }\end{array}$ & $<3,4$ à $6,>6$ ou jusqu'au noircissement \\
\hline Proportion d'huile ajoutée & $25 \%, 30 \%, 75 \%$ \\
\hline Durée de friture & 1 à $5 \mathrm{~min}, 6$ à $10 \mathrm{~min}, 11$ à $15 \mathrm{~min},>16 \mathrm{~min}$ \\
\hline Température de l'huile & $<160^{\circ} \mathrm{C}, 160-180^{\circ} \mathrm{C},>180^{\circ} \mathrm{C}$ \\
\hline Taux de composés néoformés & $<25 \%, 25-75 \%,>75 \%$ \\
\hline
\end{tabular}

\section{RESULTATS}

\section{Profil des enquêtés}

Le profil des enquêtés prend en compte l'âge des vendeurs, leurs sexes et leur niveau d'instruction. Le tableau 2 montre que les vendeurs enquêtés sont majoritairement de sexe masculin $(94,9 \%)$. Très peu de vendeurs ont un niveau d'instruction. En effet, l'enquête a révélé que $63 \%$ des vendeurs n'ont aucun niveau scolaire; mais $33,1 \%$ de cette catégorie ont fréquenté une école coranique.

\section{Pratiques de friture}

Les pratiques de friture sont présentées dans le tableau 3 ci-dessous. Il ressort que $70 \%$ des vendeurs enquêtés font jusqu'à 6 fournées de friture avec la même huile et $14,44 \%$ font la friture avec la même huile jusqu'à son noircissement total. La durée de la friture varie entre 1 et $5 \mathrm{mn}$ chez $13,33 \%$ des enquêtés, 6 et $10 \mathrm{mn}$ chez $31,11 \%, 11$ et 15 $\mathrm{mn}$ chez $46,66 \%$ et est supérieure à $16 \mathrm{mn}$ chez $8,88 \%$ des enquêtés. En outre une grande majorité de vendeurs $(76,66 \%)$ font la friture de 6 à 10 poissons par fournées tandis que $16,66 \%$ font frire 11 à 15 poissons par fournées. Concernant la température de friture, il a été constaté trois grandes tendances: $62,22 \%$ des vendeurs font la friture à des températures comprises entre 160 et $180{ }^{\circ} \mathrm{C}$; chez $24,44 \%$ des vendeurs, la température est inférieure à $160{ }^{\circ} \mathrm{C}$ et supérieure à $180 \quad{ }^{\circ} \mathrm{C}$ chez $13,33 \%$ des vendeurs.

\section{Taux des composés néoformés des huiles réutilisées}

La majorité des huiles analysées présente des teneurs en composés néoformés supérieures ou égale à $25 \%$ et réparties comme suit : $59,99 \%$ d'huiles contiennent des taux compris dans l'intervalle [25; 75\%[ et $34,44 \%$ d'huiles analysées présentent des taux supérieurs à $75 \%$. Seulement $5,55 \%$ des huiles analysées ont des taux inférieurs à 25\% (Figure 1).

\section{Caractéristiques des différentes pratiques de friture}

En se basant sur le principe de pourcentage maximal de variance cumulée, les 8 premiers axes sont retenus. Ils accumulent environ $50 \%$ de la variance totale cumulée.

De l'analyse de la Figure 2, par rapport à l'axe 1 , il ressort que les vendeurs qui se ravitaillent en huile dans les supermarchés font la friture des thons pendant 11 et 15 minutes lors des fournées jusqu'au noircissement de l'huile avec moins d'un litre 
en début de friture et font au fur et à mesure des rajouts d'huile. Les teneurs en composés néoformées (Total polar materials) dans leurs huiles après les fournées sont comprises entre 25 et $50 \%$ (axe 1). Les vendeurs de sexe féminin qui ont fréquenté l'école coranique achètent entre 6 à 9 litres d'huiles par jour au marché pour leur activité. Elles débutent la friture avec trois litres et réutilisent la même huile qui contient 25 à $50 \%$ de composés néoformés 4 à 6 fois pour la friture de 1 à 5 poissons pendant 11 et 15 minutes à des températures de $180{ }^{\circ} \mathrm{C}$ en moyenne (axe 2). Les vendeurs qui ont le niveau du primaire utilisent le bois comme source d'énergie; la quantité d'huile utilisée par ces derniers est aussi faible (moins d'un litre) par fournée. Mais, les huiles qu'ils réutilisent ont un taux de composés néoformés compris entre 50 et $75 \%$ (axe 3). On remarque aussi que les vendeurs qui ont un niveau d'étude se ravitaillent en huile dans les supermarchés. Ils réutilisent la même huile plus de 3 fois pour la friture de 11 à 15 poissons et pour d'autres, 6 à 10 poissons par fournée pendant un temps supérieur à 16 minutes (axe 4). Par contre les vendeurs ayant le niveau secondaire se ravitaillent en huile au marché pour frire les poissons. Certains parmi eux font moins de trois fournées avant le rajout d'huile. Mais d'autres utilisent l'huile plus de 6 fois ou même jusqu'à son noircissement avec 3 à 6 litres d'huiles par fournée pour frire les poissons pendant 6 à 10 minutes ou plus de 16 minutes à des températures allant de $160{ }^{\circ} \mathrm{C}$ jusqu'au-delà de $180{ }^{\circ} \mathrm{C}$. Les bains d'huiles contiennent 50 à $75 \%$ de composés néoformés (axe 5). Cependant, les vendeurs qui n'ont aucun niveau d'instruction utilisent 2 sources d'énergies (le bois et le charbon de bois) et se ravitaillent en huile chez les marchands ambulants pour la friture des thons pendant 1 à 5 minutes à des températures supérieures à $180{ }^{\circ} \mathrm{C}$ jusqu'au noircissement de l'huile. Mais, le pourcentage de composés néoformés dans leurs huiles de friture est supérieures à $75 \%$ (axe 6). Aussi, Certains vendeurs sans niveau d'instruction et certains de niveau secondaire se ravitaillent en huile de nature inconnue chez les marchands ambulants et au marché. Ils utilisent 3 à 6 litres d'huiles par fournée 4 à 6 fois. Les fritures durent 6 à 10 minutes à des températures inférieures à 160 ${ }^{\circ} \mathrm{C}$ et les teneurs en composés néoformés des huiles réutilisées sont inférieures à $25 \%$ (axe 7). Les vendeurs de sexe masculin qui ont fréquenté uniquement les écoles coraniques utilisent aussi 2 sources d'énergies (le charbon de bois et le gaz) pour frire les poissons avec des huiles d'origines inconnues durant 11 à 15 minutes à des températures supérieures à 180 ${ }^{\circ} \mathrm{C}$. Les huiles réutilisées contiennent des taux en composés néoformés supérieurs à $75 \%$. Ceux qui utilisent le charbon de bois comme source d'énergie font frire seulement 1 à 5 poissons avec 3 à 6 litres à chaque fournée. Les utilisateurs de gaz comme source d'énergie font frire 11 à 15 poissons par fournée avec une quantité de 6 à 9 litres d'huiles (axe 8).

A partir de ces résultats précédents, trois groupes stables peuvent être formés (Figures 3 et 4). Le groupe 1 renferme tous les vendeurs dont les bains d'huiles contiennent des composés néoformés inférieurs à $25 \%$. Le groupe 2 est caractérisé par une forte proportion de vendeurs dont les bains d'huiles ont un taux de composés néoformés compris entre 25 et $75 \%$. Le groupe 3 est dominé par les vendeurs dont les huiles ont des taux de composés néoformés supérieurs à $75 \%$. Les groupes 2 et 3 sont dominés par les vendeurs dont les températures de friture vont de $160 \quad{ }^{\circ} \mathrm{C}$ jusqu'au-delà de $180{ }^{\circ} \mathrm{C}$ alors que le groupe 1 est caractérisé par les vendeurs dont majoritairement les températures de la friture sont inférieures à $160{ }^{\circ} \mathrm{C}$. Dans le groupe 3 , les lots de poissons sont frits de façon continue avec rajout d'huile après moins de trois fritures. Alors que le groupe 2 comporte les vendeurs qui font la friture des lots de poissons 4 à 6 fois avec la même huile et souvent jusqu'au noircissement de l'huile. Le groupe 1 par contre contient la totalité des vendeurs qui font la friture de plusieurs lots de poissons pendant 6 à 10 minutes et $72 \%$ des vendeurs du groupe 3 entre 11 et 15 minutes. 
Tableau 2: Profil des vendeurs enquêtés.

\begin{tabular}{clc}
\hline Variables & Modalités & Pourcentages (\%) \\
\hline Ages & Jeune (15-30 ans) & 45,9 \\
& Adulte (31-50 ans) & 54,1 \\
\hline Sexes & Masculin & 94,9 \\
& Féminin & 5,1 \\
\hline Niveau & Aucun & 29,7 \\
d'instruction & Coranique & 33,1 \\
& Primaire & 14,0 \\
& Secondaire & 17,0 \\
& Supérieur & 6,4 \\
\hline
\end{tabular}

Tableau 3: Caractéristiques des pratiques de friture des vendeurs enquêtés.

\begin{tabular}{ccc}
\hline Variables & Modalités & Pourcentages (\%) \\
\hline \multirow{2}{*}{$\begin{array}{c}\text { Nombre d'utilisation de l'huile } \\
\text { pour la friture }\end{array}$} & $<3$ & 15,50 \\
& 4 à 6 & 70,00 \\
& $>6$ ou jusqu'au noircissement & 14,44 \\
Température de friture $\left({ }^{\circ} \mathrm{C}\right)$ & {$[160$ à 180$]$} & \\
& $<160$ & 62,22 \\
& $>180$ & 24,44 \\
& 1 à 5 & 13,33 \\
Durée de friture $(\mathrm{mn})$ & 6 à 10 & \\
& 11 à 15 & 13,33 \\
& $>16$ & 31,11 \\
& 16 & 8,66 \\
fournée & 6 à 10 & 8,88 \\
& 11 à 15 & 2,22 \\
& 16 à 20 & 76,66 \\
\hline
\end{tabular}


Tableau 4: Axes et modalités à interpréter.

\begin{tabular}{cc}
\hline Axes & Modalités \\
\hline Axe 1: & Supermarché, < 1L, noircis, 11 à 15min, [25; 50\%[ de composés néoformés, rajout d'huile
\end{tabular}

Axe 2: $\quad$ Coranique, Féminin, 6 à 9L, 11 à 15min, 1 à 5 Poissons, marché, 4 à 6 fois, $180^{\circ} \mathrm{C}, 25-50 \%$

Axe 3: $\quad$ Primaire, Bois, < 1L, [50; 75\%[ de composés néoformés

Axe 4: Supérieure, supermarché, < 3fois, >16min, 11 à 15 Poissons, 6 à 10 Poissons

Axe 5: $\quad$ Secondaire, marché, 3 à $6 \mathrm{~L},<3$ fois, 4 à 6 fois, noircis., >16 min, 6 à 10 min, 160 à $180^{\circ}$, $>180^{\circ},[50 ; 75 \%[$ de composés néoformés

Axe 6: Aucun, bois et charbon, marchand ambulant, noircis., 1 à 5 min, $180^{\circ} \mathrm{C},>75 \%$ composés néoformés

Axe 7: Aucun, secondaire, inconnue, marché, 3 à $6 \mathrm{~L}, 4$ à 6 fois, 6 à $10 \mathrm{~min},<160^{\circ} \mathrm{C},<25 \%$ de composés néoformés.

Axe 8: Coranique, charbon, gaz, masculin, inconnue, 3 à 6L, 6 à 9 L, 11 à 15 min, 1 à 5 Poiss, 11 à 15 Poiss, $>180^{\circ} \mathrm{C},>75 \%$ composés néoformés

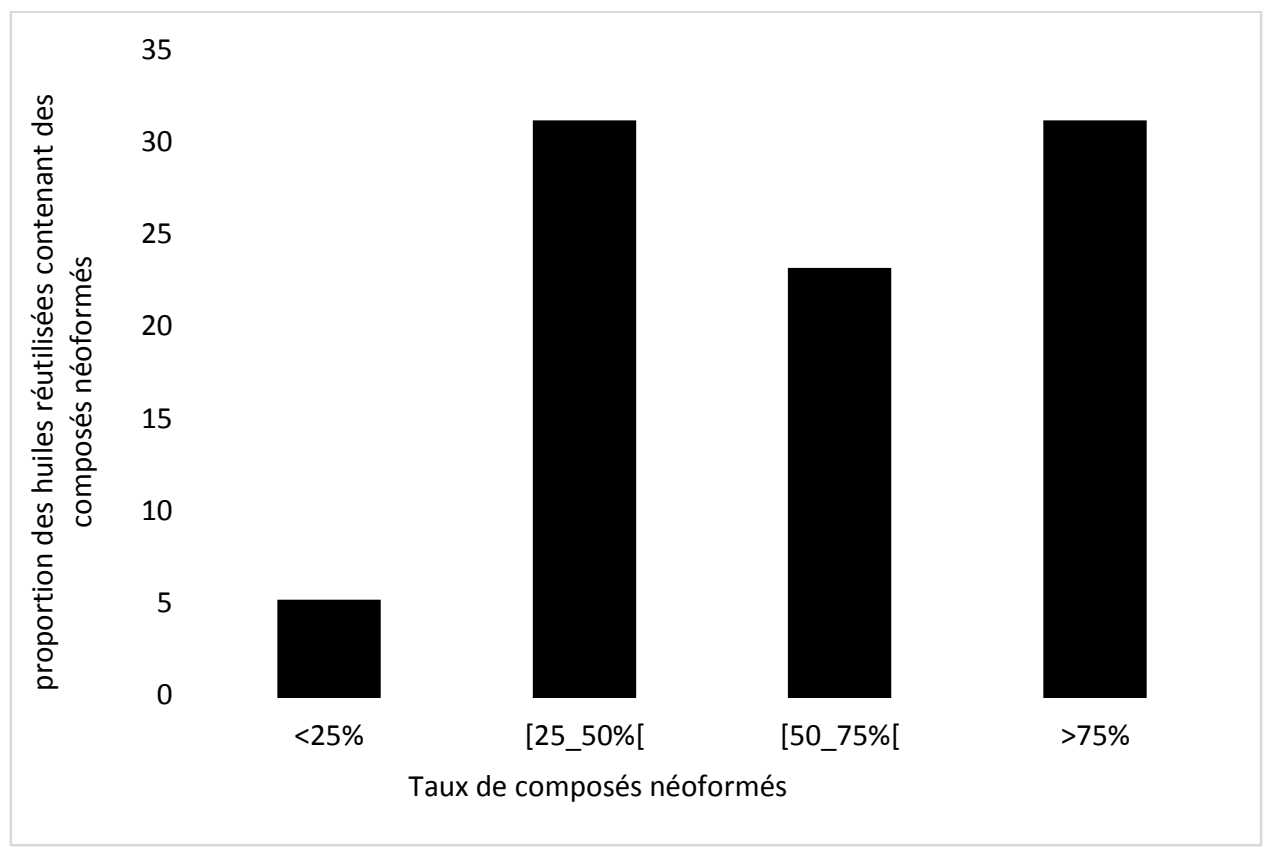

Figure 1: Diagramme du taux de composés néoformés des huiles analysées. 
M. DIABATE et al. / Int. J. Biol. Chem. Sci. 12(3): 1333-1344, 2018

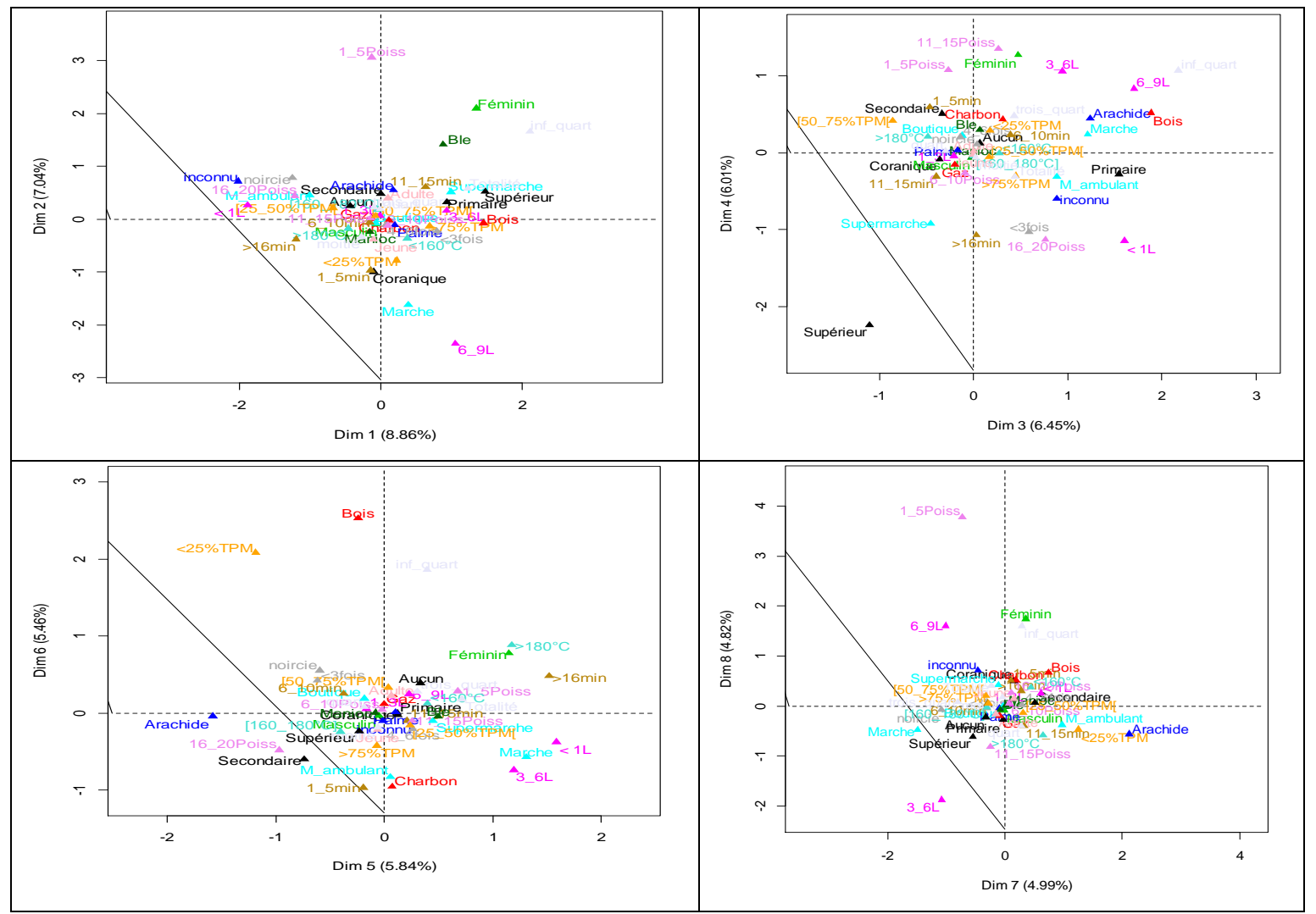

Figure 2: Caractéristiques des pratiques projetées sur les 08 axes.

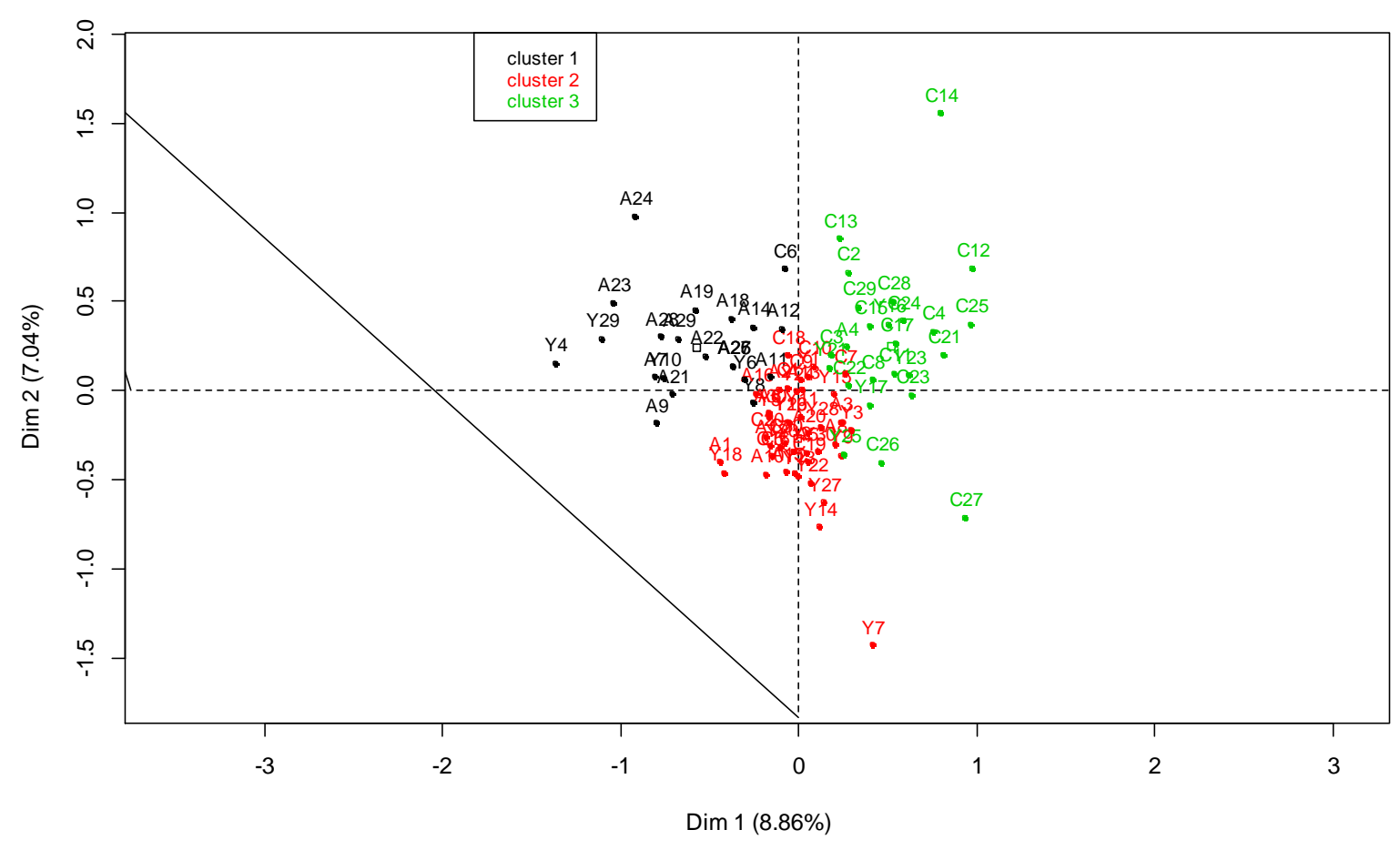

Figure 3 : Projection des groupes sur les 2 premiers axes factoriels. 


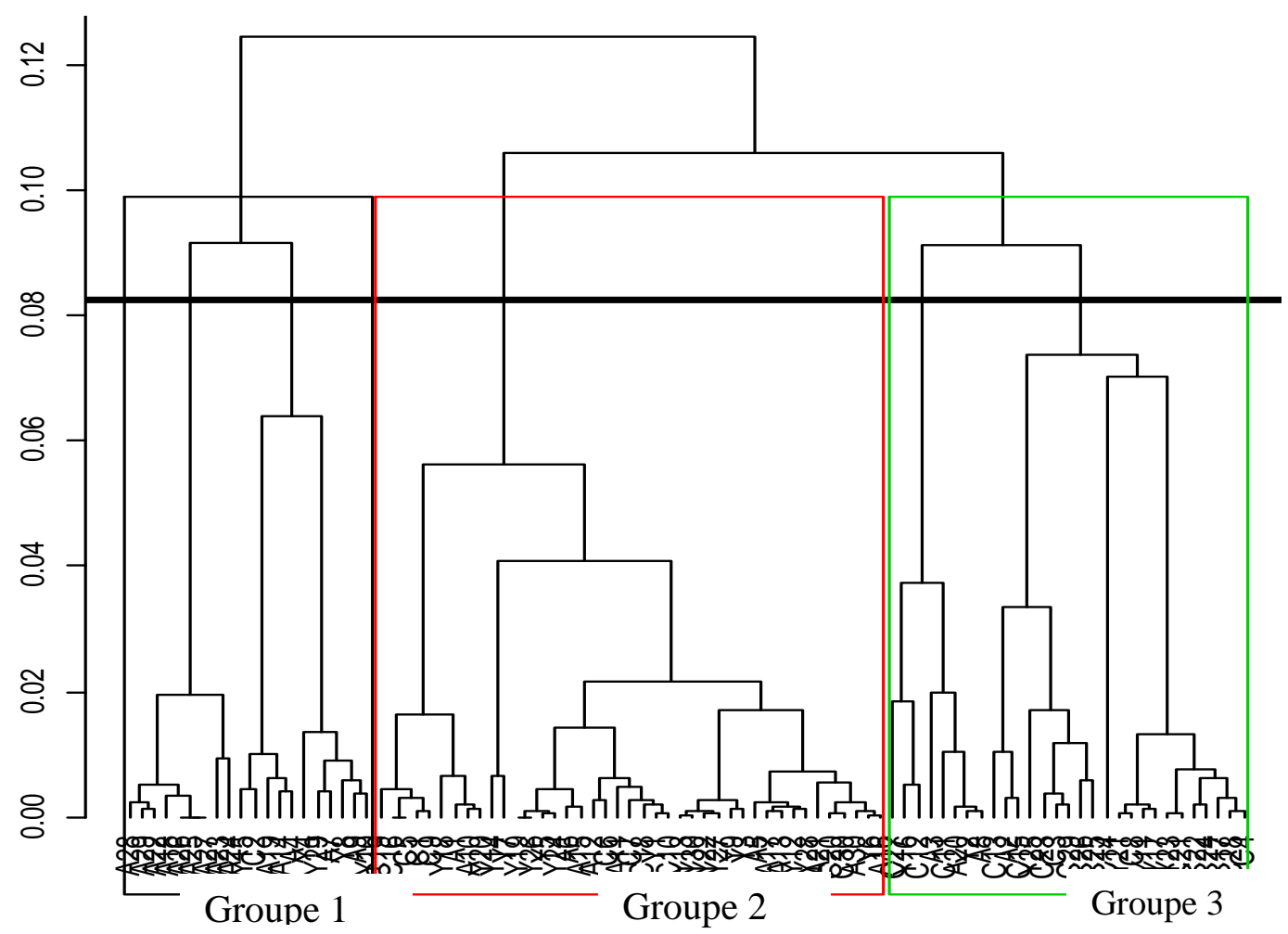

Figure 4 : Dendrogramme montrant les groupes formés.

\section{DISCUSSION}

L'activité de vente du mets «Garba» est plus effectuée par des hommes $(94,9 \%)$, ce qui viendrait du fait que l'initiateur de l'activité a été un homme qui serait d'origine nigérienne dont le patronyme fut Garba. L'intéressement des femmes $(5,1 \%)$ serait dû à l'expansion économique que connaît l'activité ces dernières années. En plus, environ $62,8 \%$ des vendeurs de ce mets n'ont aucune formation académique. Cela pourrait être dû à la forte présence dans cette activité d'hommes venus du Niger (Soungari et al., 2017), un pays où l'école coranique est privilégiée par les familles à $95 \%$ de religion musulmane (Forum Social Mondial de Tunis, 2013). Cependant, la présence de vendeurs de niveau supérieur et secondaire viendrait certainement des difficultés d'insertion des jeunes après les études.

La plupart des vendeurs utilisent pour la friture de grandes quantités d'huiles variant de 3 à 9 litres au début de l'opération. Ceci amène certains vendeurs, pour des raisons économiques, à réutiliser et même jusqu'au noircissement le bain d'huile pour la friture. Or, d'après Fang et al. (2013) l'huile utilisée plusieurs fois pour la friture se dégrade en changeant sa composition nutritionnelle, c'està-dire sa fraction lipidique, ses protéines, ses minéraux, ses vitamines et ses antioxydants. Aussi, selon Saguy et Dana (2003), la surutilisation de l'huile provoque sa dégradation par la production des composés néoformés ou polaires. Pour Oke et al. (2017), les conditions de friture, c'est à dire le chauffage intense et le refroidissement répété alternativement avec des friteuses traditionnelles, favorisent l'altération des huiles par la formation de composés néoformés indésirables, aboutissant à la formation des peroxydes au cours du refroidissement. Malheureusement, les conditions observées chez les enquêtés seraient de nature à affecter la qualité sanitaire de l'huile. Ces pratiques sont probablement liées aussi bien au niveau d'étude des vendeurs qu'à leur ignorance des phénomènes de dégradation des huiles lors de la friture. Le taux élevé de composés néoformés des huiles 
de friture du thon chez les vendeurs du mets «Garba» viendrait aussi de l'application de températures élevées de friture $\left(\right.$ de $160{ }^{\circ} \mathrm{C}$ à $180^{\circ} \mathrm{C}$ ). Les vendeurs utilisent généralement le gaz, le bois et le charbon de bois comme source d'énergie sans possibilité de maîtriser et contrôler la température dans les foyers ouverts. Ce qui engendre des températures supérieures lors de la friture des poissons thons. En plus, les températures trop élevées peuvent causer la dégradation des huiles lors de la friture et favoriser une production massive de composés néoformés (Mallika et al., 2007). En effet, Pendant les multiples chauffages à environ $180^{\circ} \mathrm{C}$ que subissent les constituants majoritaires des huiles (les acides gras), il se produit des altérations chimiques lors des réactions d'oxydation, de polymérisation et d'hydrolyse (Barrera et al., 2002) sous l'effet de la chaleur entrainant ainsi la production de composés indésirables. Ceux-ci sont les acides gras trans, les triglycérides polymérisés, l'acrylamide, les hydrocarbures (Benedito et al., 2002), qui sont des produits potentiellement cancérigènes et même neurotoxiques à une certaine dose (Dobarganes et al., 2000). Certains sont très volatils, d'autres au contraire peuvent rester dans l'huile et sont donc présents dans les aliments après la cuisson.

Par ailleurs, selon Stevens et Maier (2008) les huiles riches en acides gras polyinsaturés sont beaucoup plus sensibles aux risques d'altération par oxydation à des températures trop élevées. Csallany et al. (2015) ont trouvé un certain nombre d'aldéhydes toxiques, y compris le 4-hydroxytrans-2-nonénal et le 4-oxo-2-décénal, dans des huiles chauffées à plusieurs reprises à des températures élevées sur une longue période. Ces composés induisent la détérioration de la qualité sanitaire, nutritionnelle et sensorielle des huiles et des aliments frits, mais accroissent aussi les risques pour la santé du consommateur. Ces auteurs ont affirmé dans leur étude biomédicale que ces produits de dégradation des acides gras dans l'huile de friture sont liés à un certain nombre de cancers et des maladies neurodégénératives. Chez les adultes en bonne santé, il a été constaté une sensibilité accrue à l'oxydation dans les lipoprotéines après consommation d'huiles chauffées riches en lipides oxydés par rapport aux huiles non chauffées (Sutherland et al., 2002).

\section{Conclusion}

L'étude a montré que la majorité des vendeurs du mets «Garba» adopte des pratiques de friture du thon qui constituent une source potentielle de production de composés néoformés (polaires). Cela demeure très inquiétant, car ce mets est beaucoup consommé à Abidjan. Le taux de composés néoformés des huiles varie entre $25 \%$ à plus de $75 \%$ chez la plupart des vendeurs. Ces taux dépassent largement le taux fixé par la réglementation formelle européenne selon le décret $n^{\circ} 86-857$ du 18 juillet 1986 modifiant le décret du 11 mars 1908, portant application de la loi du $1^{\mathrm{er}}$ août 1905 relative au bon usage des matières grasses et huiles comestibles qui recommande un taux inférieur ou égal à $25 \%$ dans les graisses et huiles comestibles pour l'élaboration d'aliments.

Un risque réel pour la santé des consommateurs du mets «Garba» pourrait exister. Il est alors évident que de bonnes pratiques d'usages des huiles pour la friture du thon doivent être appliquées pour limiter les risques d'intoxications alimentaires.

\section{CONFLIT D'INTERETS}

Les auteurs déclarent qu'il n'y a aucun conflit d'intérêts.

\section{CONTRIBUTIONS DES AUTEURS}

Ce travail a été conçu et réalisé en collaboration entre cinq auteurs. Les auteurs $\mathrm{MD}$ et DN ont collecté les données de l'enquête et les échantillons sur le terrain. MD a effectué les travaux de laboratoire, la recherche de littérature et a produit un draft du manuscrit. JD, GNA et AGG ont supervisé et autorisé les analyses de l'étude. Tous les auteurs ont lu et approuvé le manuscrit final.

\section{REMERCIEMENTS}

Les auteurs adressent leurs sincères remerciements au Laboratoire de Biochimie Alimentaire et de Technologies des Produits Tropicaux (LBATPT) de l'Université Nangui Abrogoua (Côte d'Ivoire) et au Laboratoire de Bio ingénierie des Procédés Alimentaires 
(LABIOPA) de l'Université d'AbomeyCalavi (Bénin), de même qu'aux évaluateurs pour tout le suivi accordé à notre étude, la relecture et les corrections pertinentes apportées au texte.

\section{REFERENCES}

Amani NG, Kamenan A. 2003. Potentialités nutritionnelles et technologie traditionnelle de transformation des denrées amylacées en Côte d'Ivoire, $2^{\text {ème }}$ Atelier international. Voies alimentaires d'amélioration des situations nutritionnelles. Thème 3: Transformation des aliments au niveau des ménages et des communautés en vue d'améliorer la (bio) disponibilité des (micro) nutriments. 23-28 Novembre 2003, Ouagadougou, Burkina Faso, pp. 383392.

Assanvo JB, Agbo GN, Behi Y, Coulin P et Farah Z. 2002. La microflore du ferment de manioc pour la préparation de l'Attiéké Adjoukrou à Dabou (Côte d'Ivoire). Biot. Rvue. Int. Sci. V.T., $\mathrm{N}^{\circ}$ special. Acte du coloque international, centre Suisse, 286-299.

Ayawovi E, Regina K, Antoine A, Sébastien N. 2016. Towards added value attieke production in Côte d'Ivoire using bacillus spp. As starters. Turk. J. Agric. Food Sci. Techno., 4(12): 1077-1084.

Barrera AD, Ruiz-Méndez MV, Velasco J, Márquez-Ruiz G, Dobarganes MC. 2002. Loss of tocopherols and formation of degradation compounds at frying temperatures in oils differing in unsaturation degree and natural antioxidant content. J. Sci. Food Agric, 82: 1696-1702. DOI: 10.1002/jsfa.1245.

Bassa AY, Adjéhi D, Sylvain GT, Koffi MD, Bassirou B, 2016. Contamination par Bacillus cereus de l'attiéké produit dans le secteur informel au sud de la Côte d'Ivoire et gestion du risque par le réchauffage hydrothermique. Inter. $J$. Innov. App. Stud., 15(3): 637-654.

Benedito J, Mulet A, Velasco J and Dobarganes MC. 2002. Ultrasonic assessment of oil quality during frying. J. Agric. Food Chem., 50: 4531-4536. DOI: $10.1021 / \mathrm{jf020230s.}$
Choe E, and Min DB. 2007. Chemistry of Deep-Fat Frying Oils. J. food Sci., 00: 0. DOI: $10.1111 / \mathrm{j} .1750-$ 3841.2007.00352.x.

Courtois F, Mohammad Ziaiifar A, Trezzani I, Trystram G. 2012. Friture profonde : les interactions huile-produit. $O C L, \mathbf{1 9}(2)$ : 89-95. DOI: $10.1684 /$ ocl.2012.0444.

Csallany AS, Han I, Shoeman DW, Chen C, Jieyao Y. 2015. 4-Hydroxynonenal (HNE), a toxic aldehyde in french fries from fast food restaurants. J. Am. Oil. Chem. Soc., 92: 1413-1419. DOI: 10.1007/s11746-015-2699-z.

Dobarganes MC, Velasco J, and Dieffenbacher A. 2000. Determination of polar compounds, polymerized and oxidized triacylglycerols and diacylglycerols in oils and fats. P. App. Chem., 72: 1563-1575. DOI: 10.1351/pac200072081563.

Fang W, Lianzhou J, Xiuqing Z and Juncai H. 2013. Effects of frying on polar material and free fatty acids in soybean oils. Inter. J. Food Sci. Techno., 48(6): 1218-1223. DOI: $10.1111 /$ ijfs. 12080 .

FAO. 2015. Vue d'ensemble régionale de l'insécurité alimentaire en Afrique: Des perspectives plus favorables que jamais. Accra, www.fao.org/contact-us/. ISBN : 978-92-5-208781-6.

Forum Social Mondial de Tunis. 2013. La scolarisation des filles au Niger. Atélier commun commission femme de l'UGTT et SUD éducation - solidarité. www.sudeducation.org.

Heuberger C. 2005. Cyanide content of cassava and fermented product with focus on attieke and attieke Garba. PhD thesis, swiss Fedéral Institute of Technology Zurich, 126.

DOI: https://doi.org/10.3929/ethz-a005182128.

Institut national de la statistique de Côte d'Ivoire. 2014. Rapport d'activités de l'Institut national de la statistique des résultats du $4^{\text {ème }}$ Recensement Général de la Population et de l'Habitat (RGPH) de 2014. www.ins.ci.

Konan N, Gnegoury Rene D, Hervé Alain. 2007. Le débarquement des "faux thons" ou "faux poissons" au port de pêche 
d'Abidjan : phénomène en résurgence dans les données ICCAT en Côte d'Ivoire. In: Tropical tunas $=$ Thonidés tropicaux $=$ Espèces tropicales. Madrid: ICCAT, Collective Volume of Scientific, 60(1):180-184.

http://horizon.documentation.ird.fr/exl-

doc/pleins_textes/divers1402/010056276.pdf

Mallika M, Pandey MC, Jayathilakan K, Radhakrishna K, Bawa AS, 2008. Effect of fish (Catla catla) frying on the quality characteristics of sunflower oil. Food Chem., 106: 634-639. DOI: 0.1016/j.foodchem.2007.06.023.

Oke EK, Idowu MA, Sobukola OP, Adeyeye SA, Akinsola AO. 2017. Frying of Food: A critical review. J. Culry. Sci. Techno., 1542-8044.

DOI: 10.1080/15428052.2017.1333936.

Saguy I, Dana D. 2003. Integrated approach to deep fat frying: engineering, nutrition, health and consumer aspects. J. Food Eng., 56: 143-152. DOI: 10.1016/S02608774(02)00243-1.
Soungari Y, Maïmouna Y, Bébé K. 2017. Niveau d'éducation et pratiques d'hygiène alimentaire par les vendeurs de "Garba" du District d'Abidjan. Cdian. Soc. Sci., 13(11): 46-54. DOI: http://dx.doi.org/10.3968/9997.

Stevens JF, Maier CS. 2008. Acrolein: sources, metabolism, and biomolecular interactions relevant to human health and disease. Mol. Nutr. F. Res., 52(1): 7-25. DOI: $10.1002 / \mathrm{mnfr} .200700412$.

Sutherland WHF, Jong SA, Walker RJ, Williams MJA, Skeaff CM, Duncan A. 2002. Effects of meals rich in heated olive and safflower oils on oxidation of postprandial serum in healthy men. Article in Atherosclerosis, 160(1): 195$203 . \quad$ DOI: $10.1016 / \mathrm{s} 0021-$ 9150(01)00561-5.

Tanoh B. 2016. Garba Ivoire. Journal d'Afrique l'hebdo - Edition du 22-122016. Journal hebdomadaire de la presse écrite de Côte d'Ivoire. www.abidjan.net. 